\title{
INFLUENCE OF DIFFUSION ON INTERACTIONS BETWEEN MALIGNANT GLIOMAS AND IMMUNE SYSTEM
}

Abstract. We analyse the influence of diffusion and space distribution of cells in a simple model of interactions between an activated immune system and malignant gliomas, among which the most aggressive one is GBM Glioblastoma Multiforme. It turns out that diffusion cannot affect stability of spatially homogeneous steady states. This suggests that there are two possible outcomes - the solution is either attracted by the positive steady state or by the semitrivial one. The semitrivial steady state describes the healthy state, while the positive one reflects the chronic disease and typically the level of tumour cells in this state is very high, exceeding the threshold of lethal outcome. Results of numerical simulation show that the initial tumour cells distribution has an essential impact on the dynamics of the system. If the positive steady state exists, then we observe bistability and the initial distribution decides to which steady state the solution tends.

1. Introduction. In this paper we focus on the influence of diffusion and space distribution of tumour cells in a simple model of interactions between an activated immune system and malignant gliomas, among which the most aggressive one is GBM Glioblastoma Multiforme. High grade malignant gliomas also include such tumour types as anaplastic astrocytoma and anaplastic oligodendroglioma. The life expectancy for MGs does not exceed 1.5 years for GBM and 5 years for less aggressive forms [10. Conventional treatments (surgery, radiation and chemotherapy) turn out to be insufficient in the case of MGs. Therefore, we consider immunotherapy, which can offer a survival advantage.

2010 Mathematics Subject Classification: 35K57, 35B09, 35B30, 35B35, 92D25.

Key words and phrases: reaction-diffusion equations, immune system, glioma, Glioblastoma Multiforme. 
In this paper we follow the ideas of immunotherapy presented in 14 and extended in [11]-[13]. However, following [8], in the present work we include the influence of spatial effects on the system dynamics using reactiondiffusion equations instead of ODEs. We compare the dynamics of solutions for two types of gliomas: grade III which are less aggressive and grade IV which are the most aggressive ones. In [8] we considered the diffusion process in 1-D, for simplicity. The present paper extends the results of 8 to 2-D and 3 -D cases which are closer to real experiments $(2-\mathrm{D}$ in vitro and $3-\mathrm{D}$ in vivo).

Interactions between the immune system and tumour/cancer cells and immunotherapy of cancer are currently among the most intensively studied topics, both from the medical and mathematical point of view (cf., e.g., [1], [3]-[6], [9], [15]-[18] and [22]-[26]).

1.1. Presentation of the model. The full model of immune reactions proposed in [14] and generalised in [11, 12] is described by the system of six equations, which reflect the dynamics of concentrations of the main components of the analysed processes, namely $T(t)$ reflecting the tumour size, $C(t)$ describing the number of cytotoxic T-lymphocytes (CTL), $F_{\beta}$ and $F_{\gamma}$ are the concentrations of TGF- $\beta$ and INF- $\gamma$, respectively, and MI and MII represent molecules of MHC class I and II, respectively. This system of equations reads

$$
\begin{aligned}
\dot{T} & =r(T) T-f_{T}\left(F_{\beta}\right) g_{T}(M I) h(T) C T, \\
\dot{C} & =f_{C}(T \cdot M I I) g_{C}\left(F_{\beta}\right)-\mu_{C} C+S(t), \\
\dot{F}_{\beta} & =f_{\beta}(T)-\mu_{\beta} F_{\beta}, \\
\dot{F}_{\gamma} & =f_{\gamma}(C)-\mu_{\gamma} F_{\gamma}, \\
\dot{M} I & =f_{M I}\left(F_{\gamma}\right)-\mu_{M I} M I, \\
\dot{M} I I & =f_{M I I}\left(F_{\beta}\right) g_{M I I}\left(F_{\gamma}\right)-\mu_{M I I} M I I,
\end{aligned}
$$

where:

- $r(T)$ is the rate of tumour growth; it is at most linear, i.e. $r(T) \leq r_{0}$, $r_{0}=$ const, and $r(K)=0$, where $K$ reflects the maximal tumour size (in the following we study the model with $r(T)=r(1-T / K)$ );

- $f_{T}\left(F_{\beta}\right)$ and $g_{T}(M I)$ describe the CTL efficiency; $f_{T}$ is decreasing and bounded from below, $g_{T}$ is increasing and bounded from above;

- $h(T)$ is the tumour damping function; it is decreasing and bounded from below;

- $f_{C}(T \cdot M I I)$ is the CTL production with the inhibition rate $g_{C}\left(F_{\beta}\right)$ depending on TGF- $\beta ; f_{C}(T \cdot M I I)$ is increasing and bounded from above, while $g_{C}\left(F_{\beta}\right)$ is decreasing and bounded from below; 
- $S(t)$ is the treatment function, that is, the infusion of alloreactive CTLs; in general $S(t)$ is non-negative and bounded from above; we focus on the case $S(t)=S=$ const;

- $f_{\beta}(T)$ and $f_{\gamma}(C)$ are the production functions of TGF- $\beta$ and IFN- $\gamma$, respectively; $f_{\beta}(T)$ and $f_{\gamma}(C)$ are increasing;

- $f_{M I}\left(F_{\gamma}\right)$ is the MHC class I production function; it is increasing and bounded from above;

- $g_{M I I}\left(F_{\gamma}\right)$ and $f_{M I I}\left(F_{\beta}\right)$ are the MHC class II production and inhibition, respectively; $g_{M I I}\left(F_{\gamma}\right)$ is increasing and bounded from above, while $f_{M I I}\left(F_{\beta}\right)$ is decreasing and bounded from below.

We also assume that all the functions described above are at least of class $\mathbf{C}^{1}$.

Following the ideas presented in [1]-13] we simplify the notation using the following new variables: $x=F_{\beta}, y=F_{\gamma}, u=M I, v=M I I$, and function's indices: $f_{\beta}=f_{x}, f_{\gamma}=f_{y}, f_{M I}=f_{u}, f_{M I I}=f_{v}$ and $g_{M I I}=g_{v}$. Moreover, as in [13] we assume that all functions are strictly convex, concave or linear, more precisely:

- $r(T)$ is non-increasing and convex or linear,

- $f_{T}$ is convexly decreasing from $f_{T}(0)=1$ to $1>a_{T, x}>0$,

- $g_{T}$ is concavely increasing from $g_{T}(0)=0$ to $a_{T}>0$,

- $h$ is convexly decreasing from $h(0)=1$ to 0 ,

- $f_{C}$ is concavely increasing from $f_{C}(0)=0$ to $a_{C, v}>0$,

- $g_{C}$ is convexly decreasing from $g_{C}(0)=1$ to $1>a_{C, x} / e_{C, x}>0$,

- $f_{x}$ and $f_{y}$ are increasing and concave or linear, $f_{x}(0)=g_{x}>0$ and $f_{y}(0)=0$, respectively,

- $f_{u}$ is concavely increasing from $f_{u}(0)=g_{y}>0$ to $g_{y}+a_{u, y}>g_{y}$,

- $f_{v}$ is convexly decreasing from $f_{v}(0)=1$ to 0 and finally,

- $g_{v}$ is concavely increasing from $g_{v}(0)=0$ to $a_{v, y}>0$.

The simplified model proposed in [1] assumes that the dynamics of TGF- $\beta$ is much faster than those of other system components, which is based on the turnover and secretion rate estimated for TGF- $\beta$ from experimental data. Therefore, the variable $x$ is determined as $x=f_{x}(T) / \mu_{x}$. Furthermore, we neglect the influence of MHC class II receptors and TGF- $\beta$ on the natural CTLs inflow and assume that the inflow of CTLs is constant. Define the auxiliary function

$$
\bar{f}_{T}(T)=f_{T}\left(f_{x}(T) / \mu_{x}\right) .
$$

Assuming further that all components of the system have some spatial distribution, that is, $T=T(t, p), C=C(t, p), y=y(t, p), u=u(t, p), p \in \mathbb{R}^{3}$, and they can diffuse in space with constant diffusion coefficients, the final 
version of the system studied in this paper reads

$$
\begin{aligned}
& \frac{\partial T}{\partial t}=r(T) T-\bar{f}_{T}(T) g_{T}(u) h(T) T C+D_{T} \Delta T \\
& \frac{\partial C}{\partial t}=S-\mu_{C} C+D_{C} \Delta C \\
& \frac{\partial y}{\partial t}=f_{y}(C)-\mu_{y} y+D_{y} \Delta y \\
& \frac{\partial u}{\partial t}=f_{u}(y)-\mu_{u} u+D_{u} \Delta u
\end{aligned}
$$

with the standard Neumann boundary conditions on $[0, \pi]^{3} \subset \mathbb{R}^{3}$, for simplicity.

We would like to study the dynamics of tumour growth for gliomas of grade III and IV. Note that the form of equations is the same for both grades of tumour and the difference is expressed by the value of the reproduction coefficient which is much greater for grade IV than for grade III. On the basis of numerical simulations in Section 3 we show that the qualitative behaviour for both grades is similar. However, there are major quantitative differences.

2. Properties of the system. In [11, 12 the basic properties of the full system (1) were studied. It was proved that in the case of tumour growth, that is, for $T \in[0, K]$, all variables are bounded and this leads to dissipativity of the system. Dissipativity implies that the system (1) has a compact global attractor. In all cases considered in [12] the solution was attracted by one of the steady states.

2.1. Existence of solutions. Local existence of solutions to $(2)$ is an easy consequence of the theory set out in [19]. To prove global existence we show that the system has an invariant set. Note that the same set is invariant for the system without diffusion (cf. [12]). We use the framework of invariant sets presented in [5, 20, 21].

Proposition 1. The set $\boldsymbol{\Sigma}=[0,1] \times\left[0, C^{*}\right] \times\left[0, y^{*}\right] \times\left[0, u^{*}\right]$, where $C^{*}=S / \mu_{C}, y^{*}=f_{y}\left(C^{*}\right) / \mu_{y}$ and $u^{*}=f_{u}\left(y_{m}\right) / \mu_{u}$, is invariant for (2).

Proof. Write $(T, C, y, u)=Y=\left(Y_{1}, Y_{2}, Y_{3}, Y_{4}\right)$. Let $\Phi(Y)$ be the vector of kinetic functions of (2). Following the ideas of [20] we look for a function $G: \mathbb{R}^{4} \rightarrow \mathbb{R}$ such that $G(Y) \leq 0$ in $\boldsymbol{\Sigma}, G$ is quasi-convex, $\nabla G$ is a left eigenvector for the diffusion coefficients matrix $D\left(D=\operatorname{diag}\left(D_{Y_{i}}\right)\right.$ in our case), and $\left.\nabla G \cdot \Phi\right|_{\mathrm{bd} \boldsymbol{\Sigma}} \leq 0$.

Since the matrix $D$ is diagonal, every vector $S^{l} \in \mathbb{R}^{4}$ with $S_{i}^{l}=0$ for $i \neq l$ and $S_{l}^{l}= \pm 1$ is a left eigenvector for this matrix. Therefore, we can use functions $G_{i}$ which are linear as functions of $Y_{i}$. 
To show non-negativity we use the functions $G_{i}=-Y_{i} \leq 0$ in $\boldsymbol{\Sigma}, i=$ $1,2,3,4$. We have

$$
\begin{aligned}
& \nabla G_{1}(Y) \cdot \Phi(Y)=-\left.T\left(r(T)-\bar{f}_{T}(T) g_{T}(u) h(T) C\right)\right|_{T=0}=0, \\
& \nabla G_{2}(Y) \cdot \Phi(Y)=-\left.\left(S-\mu_{C} C\right)\right|_{C=0}=-S \leq 0, \\
& \nabla G_{3}(Y) \cdot \Phi(Y)=-\left.\left(f_{y}(C)-\mu_{y} y\right)\right|_{y=0}=-f_{y}(C) \leq 0, \\
& \nabla G_{4}(Y) \cdot \Phi(Y)=-\left.\left(f_{u}(y)-\mu_{u} u\right)\right|_{u=0}=-f_{u}(y) \leq 0 .
\end{aligned}
$$

Next we use $G_{i}(Y)=Y_{i}-Y_{i}^{m} \leq 0$ in $\boldsymbol{\Sigma}$, where $Y_{1}^{m}=1, Y_{2}^{m}=C^{*}, Y_{3}^{m}=y^{*}$ and $Y_{4}^{m}=u^{*}$. For these functions we obtain

$$
\begin{aligned}
\nabla G_{1}(Y) \cdot \Phi(Y) & =\left.\left(r(T)-\bar{f}_{T}(T) g_{T}(u) h(T) C\right)\right|_{T=1} \\
& =-\bar{f}_{T}(1) g_{T}(u) h(T) C \leq 0, \\
\nabla G_{2}(Y) \cdot \Phi(Y) & =\left.\left(S-\mu_{C} C\right)\right|_{C=C^{*}}=0, \\
\nabla G_{3}(Y) \cdot \Phi(Y) & =\left.\left(f_{y}(C)-\mu_{y} y\right)\right|_{y=y^{*}}=f_{y}(C)-f_{y}\left(C^{*}\right) \leq 0, \\
\nabla G_{4}(Y) \cdot \Phi(Y) & =\left.\left(f_{u}(y)-\mu_{u} u\right)\right|_{u=u^{*}}=f_{u}(y)-f_{u}\left(y_{m}\right) \leq 0,
\end{aligned}
$$

because $f_{y}$ and $f_{u}$ are increasing.

Therefore, $\boldsymbol{\Sigma}$ is invariant according to the theory of invariant sets for reaction-diffusion equations.

The statement of Proposition 1 implies that the solutions to (2) for initial conditions having values in $\boldsymbol{\Sigma}$ are global in time.

In the following we study (2) in the invariant domain $\boldsymbol{\Sigma}$.

2.2. Local stability analysis. Global analysis of stability for the system (2) without diffusion was performed in [11]. In the case without external inflow of CTLs, that is, for $S=0$, the solution always tends to the semitrivial steady state with maximal tumour size $T=K$. When the external inflow $S$ is non-zero, we have two possibilities depending on the magnitude of $S$ and the initial tumour size $T_{0}$ :

I. a solution tends to one of the positive steady states if $S<S_{\min }$, where $S_{\min }$ is some threshold value, or $S \in\left(S_{\min }, S_{\mathrm{thr}}\right)$, where $S_{\mathrm{thr}}>S_{\mathrm{min}}$ is the next threshold, and the initial tumour size $T_{0}$ is sufficiently large,

II. a solution tends to the semitrivial steady state with zero tumour size if $S>S_{\mathrm{thr}}$ or $S \in\left(S_{\mathrm{min}}, S_{\mathrm{thr}}\right)$ and $T_{0}$ is sufficiently small.

In this subsection we focus on the influence of diffusion on the local stability of steady states. Spatially homogeneous steady states for the last three equations can be easily calculated to be $C^{*}=S / \mu_{C}, y^{*}=f_{y}\left(C^{*}\right) / \mu_{y}$ and $u^{*}=f_{u}\left(y^{*}\right) / \mu_{u}$, respectively. For the first equation the steady state value $T^{*}$ is given either by $T=0$, or by

$$
r(T)-\bar{f}_{T}(T) g_{T}\left(u^{*}\right) h(T) C^{*}=0 .
$$


This can be reformulated as

$$
r(T)=G(T) H(S)
$$

where

$$
G(T)=f_{T}\left(\frac{f_{x}(T)}{\mu_{x}}\right) h(T), \quad H(S)=g_{T}\left(\frac{f_{u}\left(f_{y}\left(S / \mu_{C}\right)\right)}{\mu_{u}}\right) \frac{S}{\mu_{C}} .
$$

Proposition 2. The system (2) has at most two spatially homogeneous positive stationary solutions (steady states).

Proof. Positive steady states are defined by (4). For constant inflow of CTLs one needs to compare the behaviour of $r(T)$ and $G(T)$ on the interval $[0, K]$. We know that $r$ is decreasing to $r(K)=0$ and $r^{\prime \prime}(T) \geq 0$, that is, $r$ is linear or convex. We can show that $G(T)$ is convexly decreasing. In fact,

$$
G^{\prime}(T)=f_{T}^{\prime}\left(\frac{f_{x}(T)}{\mu_{x}}\right) \frac{f_{x}^{\prime}(T)}{\mu_{x}} h(T)+f_{T}\left(\frac{f_{x}(T)}{\mu_{x}}\right) h^{\prime}(T)<0,
$$

because $f_{T}$ and $h$ are decreasing, while $f_{x}$ is increasing. Moreover,

$$
\begin{aligned}
G^{\prime \prime}(T)= & f_{T}^{\prime \prime}\left(\frac{f_{x}(T)}{\mu_{x}}\right)\left(\frac{f_{x}^{\prime}(T)}{\mu_{x}}\right)^{2} h(T)+\frac{f_{x}^{\prime \prime}(T)}{\mu_{x}} f_{T}^{\prime}\left(\frac{f_{x}(T)}{\mu_{x}}\right) h(T) \\
& +h^{\prime \prime}(T) f_{T}\left(\frac{f_{x}(T)}{\mu_{x}}\right)+2 f_{T}^{\prime}\left(\frac{f_{x}(T)}{\mu_{x}}\right) \frac{f_{x}^{\prime}(T)}{\mu_{x}} h^{\prime}(T)>0,
\end{aligned}
$$

because $f_{T}$ and $h$ are convex and $f_{x}$ is linear or concave. Therefore, (4) can have at most two solutions. The number of solutions depends on the magnitude of $S$. It is easily seen that $H(S)$ is an increasing function. If $S$ is sufficiently small, we have $r(0)>G(0) H(S)$ and then there exists exactly one positive steady state. If $r(0)<G(0) H(S)$, then there are two positive steady states or none.

Studying local stability of these states for the system (2) we use the standard separation of variables method and the linearisation theorem (see e.g. 2, 20 ). Hence, we look for fundamental solutions of the form $\exp (\lambda t)$ $\times \cos \left(k_{1} p_{1}\right) \cos \left(k_{2} p_{2}\right) \cos \left(k_{3} p_{3}\right), k_{j} \in \mathbb{Z}, j=1,2,3, p=\left(p_{1}, p_{2}, p_{3}\right) \in[0, \pi]^{3}$, and obtain the following characteristic matrix:

$$
\left(\begin{array}{cccc}
a_{11}-\lambda-D_{T} K & a_{12} & 0 & a_{14} \\
0 & -\mu_{c}-\lambda-D_{C} K & 0 & 0 \\
0 & f_{y}^{\prime}\left(C^{*}\right) & -\mu_{y}-\lambda-D_{y} K & 0 \\
0 & 0 & f_{u}^{\prime}\left(y^{*}\right) & -\mu_{u}-\lambda-D_{u} K
\end{array}\right)
$$

where $K=k_{1}^{2}+k_{2}^{2}+k_{3}^{2}$, 


$$
\begin{aligned}
a_{11}= & r(T)-g_{T}(u) h(T) \bar{f}_{T}(T) C \\
& +T\left(r^{\prime}(T)-g_{T}(u) C\left(h^{\prime}(T) \bar{f}_{T}(T)+h(T) \bar{f}_{T}^{\prime}(T)\right)\right), \\
a_{12}= & -g_{T}(u) h(T) \bar{f}_{T}(T) T, \quad a_{14}=-g_{T}^{\prime}(u) h(T) \bar{f}_{T}(T) T C
\end{aligned}
$$

for $(T, C, y, u)=\left(T^{*}, C^{*}, y^{*}, u^{*}\right)$.

Proposition 3. Diffusion-driven instability cannot occur for the system (2).

Proof. The characteristic equation for both steady states has the form

$$
\begin{aligned}
\left(a_{11}\right. & \left.-\lambda-D_{T}\left(k_{1}^{2}+k_{2}^{2}+k_{3}^{2}\right)\right)\left(\mu_{C}+\lambda+D_{C}\left(k_{1}^{2}+k_{2}^{2}+k_{3}^{2}\right)\right) \\
& \times\left(\mu_{y}+\lambda+D_{y}\left(k_{1}^{2}+k_{2}^{2}+k_{3}^{2}\right)\right)\left(\mu_{u}+\lambda+D_{u}\left(k_{1}^{2}+k_{2}^{2}+k_{3}^{2}\right)\right)=0,
\end{aligned}
$$

and we look for solutions $\lambda$ that obviously depend on $k_{j}, j=1,2,3$. These solutions can be easily calculated as $\lambda_{1}=a_{11}-D_{T}\left(k_{1}^{2}+k_{2}^{2}+k_{3}^{2}\right), \lambda_{2}=$ $-\left(\mu_{C}+D_{C}\left(k_{1}^{2}+k_{2}^{2}+k_{3}^{2}\right)\right), \lambda_{3}=-\left(\mu_{y}+D_{y}\left(k_{1}^{2}+k_{2}^{2}+k_{3}^{2}\right)\right)$ and $\lambda_{4}=$ $-\left(\mu_{u}+D_{u}\left(k_{1}^{2}+k_{2}^{2}+k_{3}^{2}\right)\right)$. We see that all eigenvalues are real and $\lambda_{i}$ for $i=2,3,4$ is negative for every $k_{j} \in \mathbb{Z}, j=1,2,3$. Moreover, if $a_{11}<0$, then $\lambda_{1}<0$, while if $a_{11}>0$, then $\lambda_{1}>0$ for $k_{1}=k_{2}=k_{3}=0$. This implies that neither the trivial nor the positive steady state can lose local stability under the influence of diffusion. Clearly, if the steady state is stable for the case without diffusion, that is, $a_{11}<0$, then for any $k_{i} \in \mathbb{Z}$ all characteristic values are negative.

If

$$
S<\tilde{S}=\frac{r(0) \mu_{C}}{g_{T}\left(u^{*}\right) h(0) f_{T}(0)},
$$

then the trivial steady state is unstable independently of the magnitude of the diffusion coefficients. If $S>\tilde{S}$, then this state is stable, also independently of the diffusion coefficients. Similarly, if

$$
S<\frac{\left(r\left(T^{*}\right)+r^{\prime}\left(T^{*}\right) T^{*}\right) \mu_{C}}{g_{T}\left(u^{*}\right)\left(h\left(T^{*}\right) \bar{f}_{T}\left(T^{*}\right)+T^{*}\left(h^{\prime}\left(T^{*}\right) \bar{f}_{T}\left(T^{*}\right)+h\left(T^{*}\right) \bar{f}_{T}^{\prime}\left(T^{*}\right)\right)\right)},
$$

then the positive steady state is unstable independently of the diffusion coefficients, while for the opposite inequality it is stable. It should be noticed that the inequality above gives a condition on $S$ in an implicit formula, because $T^{*}$ is also a function of $S$. We can rewrite it in the following form:

$$
\frac{r^{\prime}\left(T^{*}\right)}{r\left(T^{*}\right)}>\frac{h^{\prime}\left(T^{*}\right)}{h\left(T^{*}\right)}+\frac{\bar{f}_{T}^{\prime}\left(T^{*}\right)}{\bar{f}_{T}\left(T^{*}\right)},
$$

noting that $a_{11}=r\left(T^{*}\right) T^{*}\left(\frac{r^{\prime}\left(T^{*}\right)}{r\left(T^{*}\right)}-\frac{h^{\prime}\left(T^{*}\right)}{h\left(T^{*}\right)}-\frac{\bar{f}_{T}^{\prime}\left(T^{*}\right)}{\bar{f}_{T}\left(T^{*}\right)}\right)$ for $T^{*}>0$. 
3. Numerical simulations. In Section 2 the analytical result was stated for the 3-D case. However, it is difficult to illustrate results of simulations in this case. Therefore, we present a simulation for $p \in[0,1]$. We use the specific realisations of the functions describing the right-hand side of the system (2) proposed in [14] and the normal values of parameters estimated in [14, 12]. Hence, after rescaling we consider the following system of equations:

$$
\begin{aligned}
\frac{\partial T}{\partial t}(t, \omega)= & d_{T} \Delta T \\
& +T\left(r(1-T)-a_{T} C \frac{u}{u+e_{T}} \frac{h_{T}}{T+h_{T}} \frac{a_{T, x} \frac{T+g_{x}}{\mu_{x}}+e_{T, x}}{\frac{T+g_{x}}{\mu_{x}}+e_{T, x}}\right),
\end{aligned}
$$

(6) $\frac{\partial C}{\partial t}(t, \omega)=d_{C} \Delta C+S_{C}-C$,

$$
\begin{aligned}
& \frac{\partial y}{\partial t}(t, \omega)=d_{y} \Delta y+C-\mu_{y} y, \\
& \frac{\partial u}{\partial t}(t, \omega)=d_{u} \Delta u+\frac{y+g_{y}}{y+e_{u, y}}-\mu_{u} u,
\end{aligned}
$$

with the standard Neumann boundary conditions on $[0,1]$ and the values of parameters:

$$
\begin{gathered}
r_{I I I}=0.0472, \quad r_{I V}=0.135, \quad a_{T}=0.208, \quad e_{T}=0.128, \quad h_{T}=5.2 \cdot 10^{-6}, \\
a_{T, x}=0.69, \quad g_{x}=0.111, \quad \mu_{x}=935, \quad e_{T, x}=1.3 \cdot 10^{-4}, \\
\mu_{y}=13.8, \quad g_{u}=0.5, \quad e_{u, y}=2.46 \cdot 10^{3}, \quad \mu_{u}=1.94, \\
S_{C}^{I V}=10^{4}, \quad S_{C}^{I I I}=3.5 \cdot 10^{3}, d_{T}=1, \quad d_{C}=d_{y}=d_{u}=0.01,
\end{gathered}
$$

where $r_{I I I}$ and $r_{I V}, S_{C}^{I I I}$ and $S_{C}^{I V}$ denote the reproductions rate and the inflows for grade $I I I$ and grade $I V$ tumour, respectively. The difference of the magnitudes of the diffusion coefficient for $T$ and for the other components of the system reflects the high diffusiveness of GBM tumour cells. In the simulations presented in [8] we used a continuous initial function and focused on the influence of the mean and maximal size of the initial tumour distribution on the tumour dynamics. It turned out that in all cases considered, at each point $p \in[0,1]$ the solution tends to one of the steady states as $t \rightarrow \infty$.

Now, we present simulations for non-standard initial functions, that is, for discontinuous values of $T_{0}$. Such initial functions seem to be more natural for experimental data, especially in vitro, when tumour cells are placed in some region at the beginning of the experiment. Other initial functions are assumed for the worst case, which means $C_{0}=y_{0}=u_{0}=0$. It should be stressed that for such type of initial functions the analytical results presented in Section 2 may be invalid. 
In the simulations we focus on the asymptotic dynamics of tumour cells as well as on the initial behaviour of the tumour which can be important from the early-treatment point of view.

We start simulations from a grade III tumour for the same initial tumour size 0.1 but with different discontinuous space distributions on subintervals of total length 0.1. The results are presented in Figs. 1 and 2, We see that independently of the number of subintervals on which the tumour cells appear and their distribution in space, the asymptotic dynamics is almost the same. Asymptotically, the number of tumour cells drops to 0, while CTLs stabilise on their steady state level $C^{*}$. Moreover, the dynamics of CTLs is exactly the same for all cases shown in Fig. 1, and therefore we present only one Fig. 2 reflecting it. However, the initial behaviour depends on the initial distribution. In Fig. 1 we see that the initial distribution of tumour cells is preserved for some time.
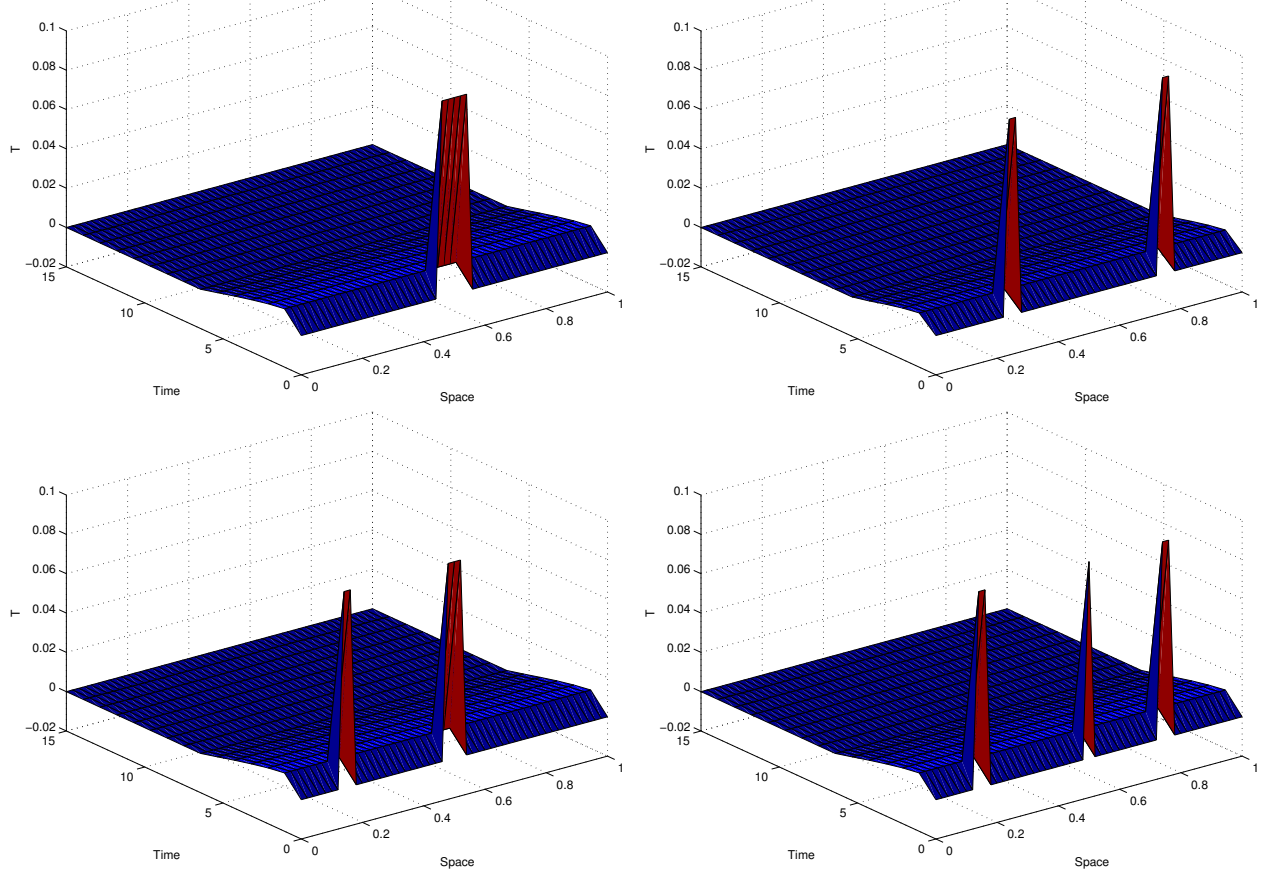

Fig. 1. Examples of tumour dynamics for a grade III tumour with initial tumour size 0.1 and different discontinuous initial tumour distributions

In Fig. 3 we see the dynamics for larger initial tumour size, namely $T_{0}=0.5$ on the interval of the same length as in Fig. 1. This is the initial level for which tumour cells stabilise at the positive steady state with increasing $t$. We see that stabilisation of CTLs on the level $C^{*}$ is very fast compared to 


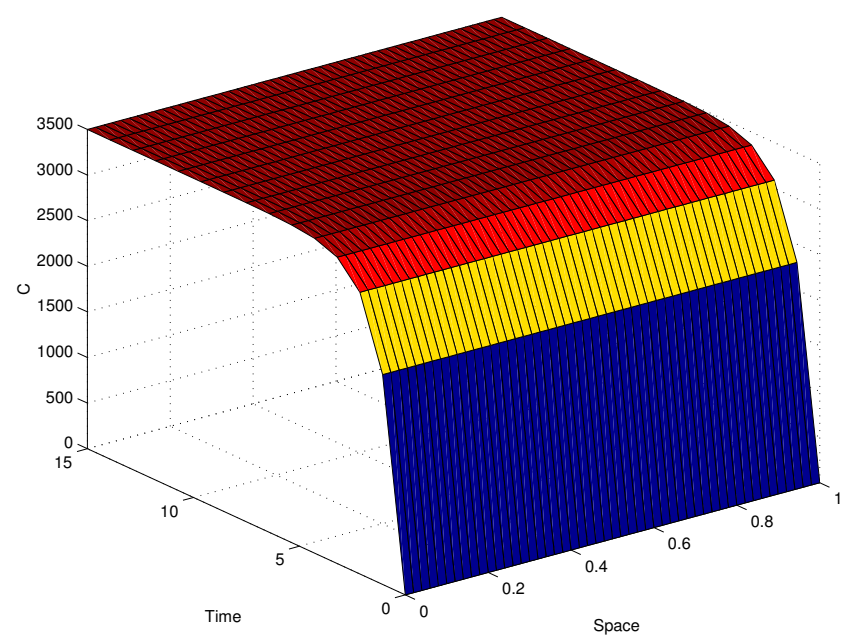

Fig. 2. The dynamics of CTLs for tumour growth presented in Fig. 1
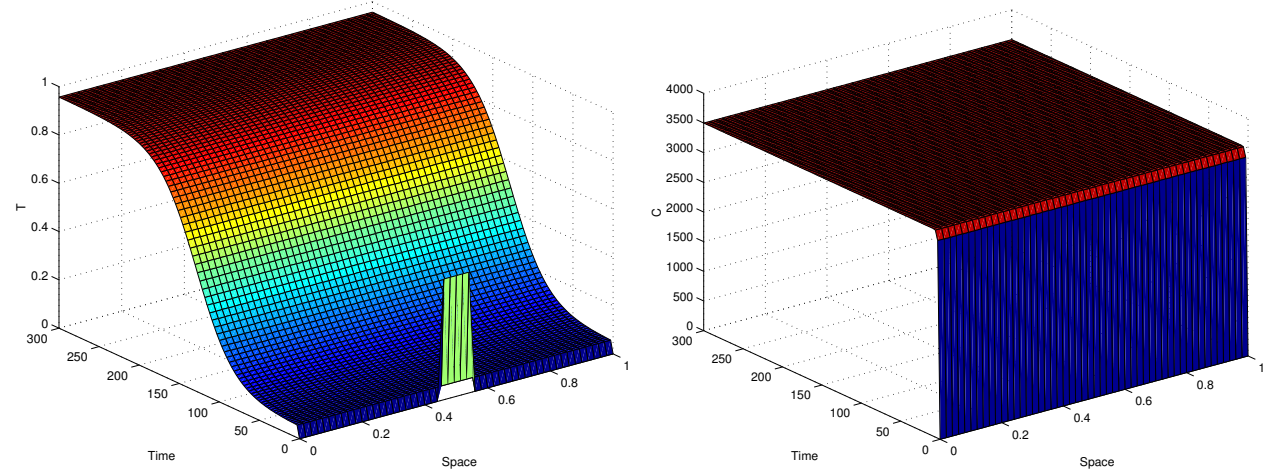

Fig. 3. Example of the tumour and CTLs dynamics for a grade III tumour with initial tumour size 0.5

the tumour growth. As before, the asymptotic dynamics does not depend on the number and space distribution of the subintervals on which tumour cells appear at the beginning of the disease. However, the initial distribution is also preserved for some time after which tumour cells spread into the whole interval $[0,1]$, reaching a much smaller level than the initial $T_{0}$.

In Figs. 4 and 5 we see that the qualitative dynamics for grade IV is similar. We observe only a quantitative difference, as in the case of simulations performed in [8]. Moreover, dependence on the distribution of tumour cells is also similar - the asymptotic behaviour of solutions depends strongly on the total length of the intervals in which $T_{0}$ is non-zero and can even be large (as in Fig. 5) if the interval is short. This means that the asymptotic tumour dynamics depends mainly on the total initial number of tumour 

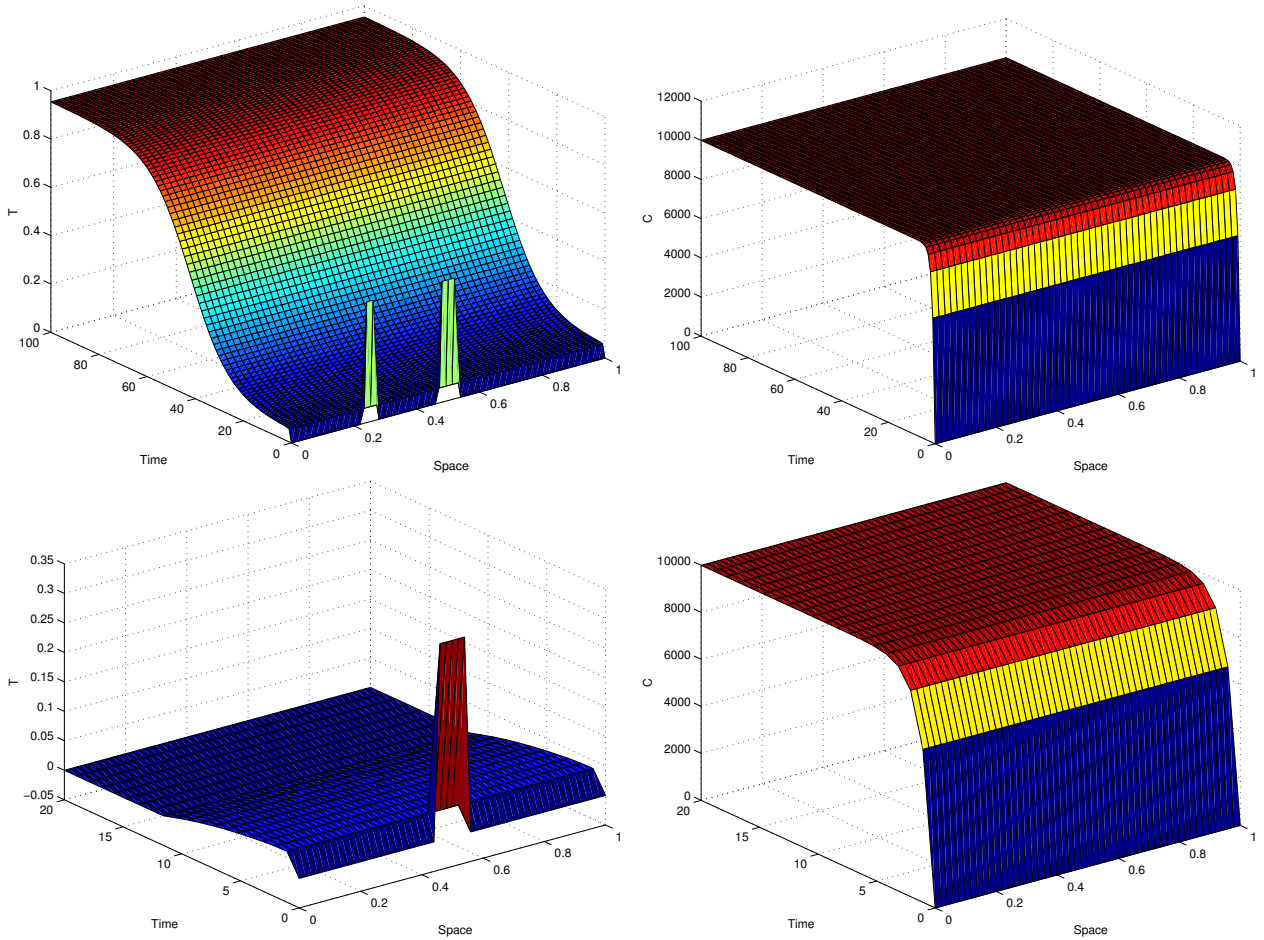

Fig. 4. Examples of the tumour and CTLs dynamics for a grade IV tumour with initial tumour size 0.5 (top) and 0.3(3) (bottom)
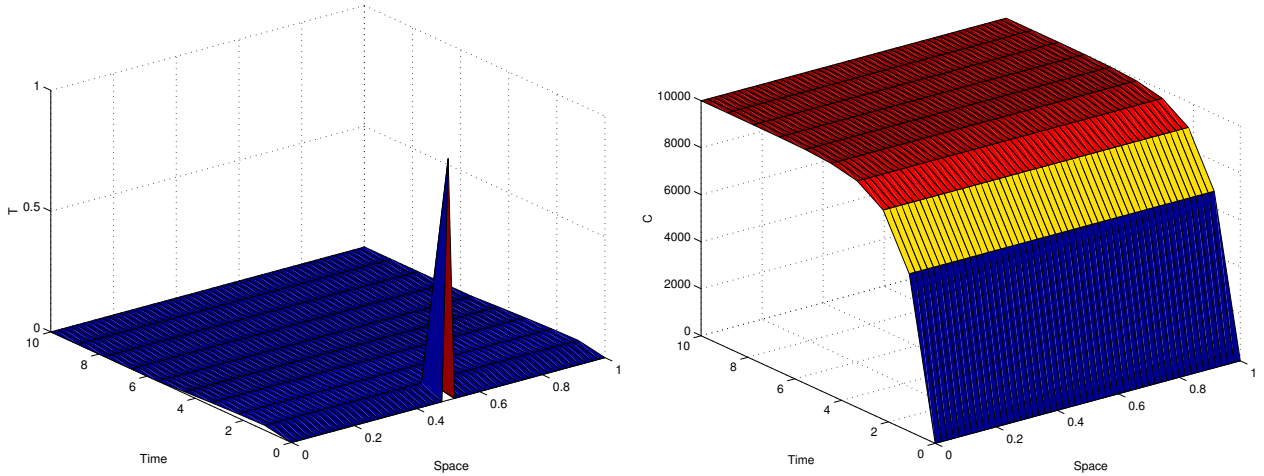

Fig. 5. Example of tumour and CTLs dynamics for a grade IV tumour with the maximal possible initial tumour size 1 on the interval of length 0.025

cells. On the other hand, for all simulations performed, we observed that the initial distribution of tumour cells is preserved for some time and then tumour cells spread either on the whole interval or on some subinterval (cf. Fig. 6). 

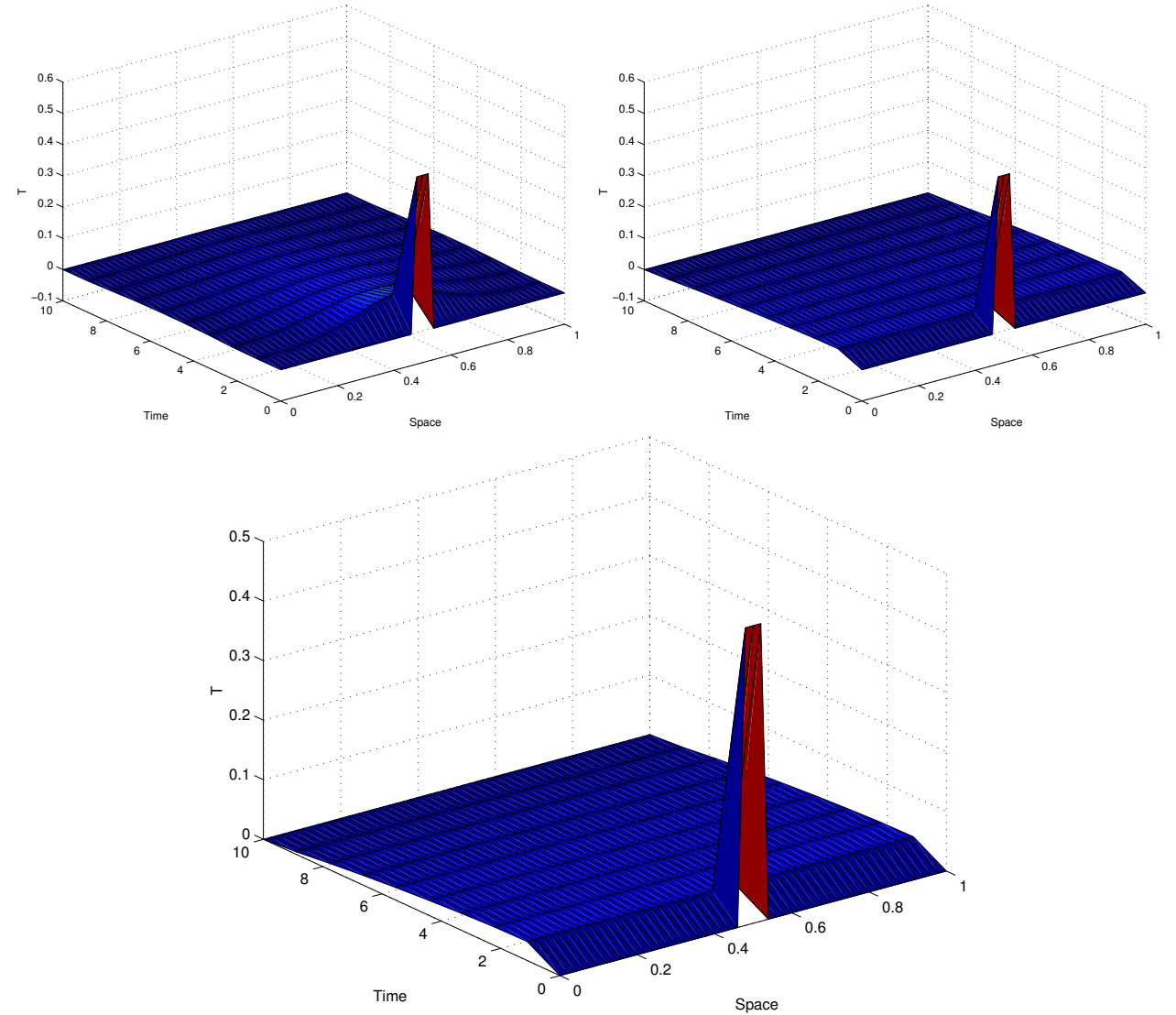

Fig. 6. Examples of tumour dynamics for a grade IV tumour and different diffusion coefficients: $0.01,1$ (top) and 100 (bottom)

We have also performed simulations for different diffusion coefficients for tumour cells (cf. Fig. 6). It is interesting that for discontinuous initial tumour distributions considered in this paper the difference in tumour cells dynamics for different diffusion coefficients is more visible than in the case of continuous functions used in [8]. For small values of the diffusion coefficient the region where tumour cells appear does not propagate into the whole interval $[0,1]$ (the upper left graph in Fig. 6), while for large diffusion coefficients it propagates very fast (the bottom graph in Fig. 6).

4. Conclusions. We have studied a simple model of immunotherapy in the case of malignant gliomas with spatial distribution reflected in the diffusion process. We have shown global existence of solutions using the framework of invariant sets, and studied local stability of steady states in the context of possible diffusion-driven instability. It turns out that diffusion 
has no essential impact on the asymptotic dynamics of the system. Stability analysis performed in Section 2 implies that stability does not change due to the diffusion process. On the other hand, from the early-treatment point of view not only the asymptotic behaviour is important. Therefore, we have performed a number of numerical simulations for different initial distributions of tumour cells assuming discontinuous initial data that seem to better reflect experimental distribution.

Numerical simulations presented in Section 3 confirmed expected bistable asymptotic dynamics - depending on the total initial tumour size, the population of tumour cells stabilises either on the zero level, which reflects recovery, or on the positive level, which typically reflects lethal outcome (this positive steady state for tumour size is normally too high for the patient's survival). On the other hand, the initial dynamics of tumour essentially depends on the initial distribution of tumour cells and on the value of the diffusion coefficient for these cells. We observe that the initial distribution is preserved for some time after which tumour cells spread either into the whole interval $[0,1]$ or to some subinterval which depends on the magnitude of diffusion. If the diffusion coefficient is large, then tumour cells occupy the whole interval and they spread into it faster with increasing diffusion. With the decreasing diffusion coefficient the interval occupied by tumour cells is smaller.

The results presented in this paper and in [8] show that although we should expect bistable asymptotic dynamics in the case of tumour-immune system interactions independently of the initial spatial tumour distribution, this initial distribution has a large impact on the initial tumour growth, which is important from the treatment point of view.

\section{References}

[1] J. C. Arciero, T. L. Jackson and D. E. Kirschner, A mathematical model of tumorimmune evasion and siRNA treatment, Discrete Contin. Dynam. Systems Ser. B 4 (2004), 39-58.

[2] N. F. Britton, Reaction-Diffusion Equations and their Application to Biology, Academic Press, New York, 1986.

[3] A. Cappuccio, M. Elishmereni and Z. Agur, Cancer immunotherapy by interleukin21 potential treatment strategies evaluated in a mathematical model, Cancer Res. 66 (2006), 7293-7300.

[4] P. A. Carpentier, W. S. Begolka, J. K. Olson, A. Elhofy, W. J. Karpus and S. D. Miller, Differential activation of astrocytes by innate and adaptive immune stimuli, Glia 49 (2005), 360-374.

[5] K. Chueh, C. Conley and J. Smoller, Positively invariant regions for systems of non-linear diffusion equations, Indiana Univ. Math. J. 26 (1977), 353-364.

[6] L. G. de Pillis, W. Gu and A. E. Radunskaya, Mixed immunotherapy and chemotherapy of tumors: modeling, applications and biological interpretations, J. Theor. Biol. 238 (2006), 841-862. 
[7] L. G. de Pillis, A. E. Radunskaya and C. L. Wiseman, A validated mathematical model of cell-mediated immune response to tumor growth, Cancer Res. 65 (2005), 7950-7958.

[8] U. Foryś, Spatial effects on interactions between brain tumour and immune systeminfluence of diffusion, in: Proc. 14th National Conference on Application of Mathematics in Biology and Medicine (Leszno n. Warsaw), M. Bodnar and U. Foryś (eds.), Univ. of Warsaw, 2008, 45-50.

[9] D. Kirschner and J. C. Panetta, Modelling immunotherapy of the tumor-immune interaction, J. Math. Biol. 37 (1998), 235-252.

[10] P. Kleihues, F. Soylemazoglu, B. Schäuble, B. W. Schniethauer and P. C. Bruger, Histopathology, classification, and grading of gliomas, Glia 15 (1995), 211-221.

[11] Y. Kogan, U. Foryś and N. Kronik, Analysis of the immunotherapy model for glioblastoma multiforme brain tumour, preprint, Institute of Applied Mathematics and Mechanics UW, 178, 2008.

[12] Y. Kogan, U. Foryś, O. Shukron, N. Kronik and Z. Agur, Cellular immunotherapy for high grade gliomas: mathematical analysis deriving efficacious infusion rates based on patient requirements, SIAM J. Appl. Math., to appear.

[13] - , - - , - , - Cellular immunotherapy for high grade gliomas: mathematical analysis of simplified model, in preparation.

[14] N. Kronik, Y. Kogan, V. Vainstein and Z. Agur, Improving alloreactive CTL immunotherapy for malignant gliomas using a simulation model of their interactive dynamics, Cancer Immunol. Immunother., to appear.

[15] C. A. Kruse, L. Cepeda, B. Owens, S. D. Johnson, J. Stears and K. O. Lillehei, Treatment of recurrent glioma with intracavitary alloreactive cytotoxic T lymphocytes and Interleukin-2, Cancer Immunol. Immunother. 45 (1997), 77-87.

[16] C. A. Kruse and D. Rubinstein, Cytotoxic T-lymphocytes reactive to patient major histocompatibility complex proteins for therapy of brain tumors, in: L. M. Liau et al. (eds.), Brain Tumor Immunotherapy, Humana Press, Totowa, NJ, 2001, 149-170.

[17] V. A. Kuznetzov, I. A. Makalkin, M. A. Taylor and A. S. Perelson, Nonlinear dynamics of immunologenic tumors: parameters estimation and global bifurcation analysis, Bull. Math. Biol. 56 (1994), 295-321.

[18] M. A. Proescholdt, M. J. Merrill, B. Ikejiri, S. Walbridge, A. Akbasak, S. Jacobson and E. H. Oldfield, Site-specific immune response to implanted gliomas, J. Neurosurg. 95 (2001), 1012-1019.

[19] F. Rothe, Global Solutions of Reaction-Diffusion Systems, Springer, 1984.

[20] J. Smoller, Shock Waves and Reaction Diffusion-Equations, Springer, New York, 1994.

[21] J. Smoller, C. C. Conley and K. N. Chueh, Positively invariant regions for systems of nonlinear diffusion equations, Indiana Univ. Math. J. 26 (1977), 373-392.

[22] J. M. Soos, J. I. Krieger, O. Stuve, C. L. King, J. C. Patarroyo, K. Aldape, K. Wosik, A. J. Slavin, P. A. Nelson, J. P. Antel and S. S. Zamvil, Malignant glioma cells use $M H C$ class II transactivator (CIITA) promoters III and IV to direct IFN- $\gamma$-inducible CIITA expression and can function as nonprofessional antigen presenting cells in endocytic processing and CD4+ T-cell activation, Glia 36 (2001), 391-405.

[23] H. M. Strik, M. Stoll and R. Meyermann, Immune cell infiltration of intrinsic and metastatic intracranial tumors, Anticancer Res. 24 (2004), 37-42.

[24] A. Suzumura, M. Sawada, H. Yamamoto and T. Marunouchi, Transforming growth factor- $\beta$ suppresses activation and proliferation of microglia in vitro, J. Immunol. 151 (1993), 2150-2158. 
[25] D. A. Thomas and J. Massagué, TGF- $\beta$ directly targets cytotoxic $T$ cell functions during tumor evasion of immune surveillance, Cancer Cell 8 (2005), 369-380.

[26] I. Yang, T. J. Kremen, A. J. Giovannone, E. Paik, S. K. Odesa, R. M. Prins and L. M. Liau, Modulation of major histocompatibility complex Class I molecules and major histocompatibility complex-bound immunogenic peptides induced by interferon $\alpha$ and interferon $\gamma$ treatment of human glioblastoma multiforme, J. Neurosurg. 100 (2004), 310-319.

Urszula Foryś

Institute of Applied Mathematics and Mechanics

Faculty of Mathematics, Informatics and Mechanics

University of Warsaw

Banacha 2

02-097 Warszawa, Poland

E-mail: urszula@mimuw.edu.pl

Received on 20.3.2009;

revised version on 24.7.2009 
